\title{
Deterrência à alimentação e à oviposição de Sitophilus zeamais Motschulsky (Coleoptera: Curculionidae) pelo uso pós vegetais em milho armazenado
}

\author{
Gustavo Soares Wenneck, Reni Saath, Larissa Leite de Araújo, Nathália de Oliveira Sá, Pedro Paschoalin
} Ramos

Universidade Estadual de Maringá - UEM, Maringá, PR. E-mail: gustavowenneck@gmail.com

\begin{abstract}
Resumo
Plantas com efeito inseticida apresentam-se entre as alternativas como ferramenta de controle dos insetospraga de grãos armazenados. No presente estudo, objetivou-se caracterizar a eficiência dos pós de origem vegetal sobre Sitophilus zeamais em grãos armazenados. Foram avaliados 14 pós vegetais (Arnica montana Baccharis trimera, Coriandrum sativum, Coffea arabica, Cymbopogon martini, Helianthus annuus, Lippia gracillis, Mentha pulegium, Moringa oleifera, Nicotiana tabacum, Piper aduncum, Piper hispidinervum, Sesamum indicum e Vitis labrusca) obtidos de material vegetal triturado, e comparado com um tratamento controle. $O$ experimento foi realizado em delineamento inteiramente casualizado, com seis repetições cada tratamento. Nos testes, $0,5 \mathrm{~g}$ dos pós inertes foram misturados aos grãos (50 g) de milho híbrido AS 1551 dentro de frascos plásticos, sendo infestados com 10 S. zeamais adultos emergidos. Após 20 dias da infestação avaliou-se a oviposição e iniciou-se a contagem de adultos emergidos. Ao fim do período de infestação, foi avaliado o peso seco dos insetos, o peso de grãos consumidos e o período de desenvolvimento (ovo-adulto). A maioria dos pós vegetais apresentou atuação efetiva sobre os insetos emergidos. Constatou-se nos pós testados redução na oviposição, exceto Vitis labrusca e Sesamum indicum cujo número de ovos foi superior ao controle, também apresentaram menor efeito sobre o desenvolvimento das larvas do caruncho. $O$ baixo peso de adultos provenientes de grãos de milho tratados com pó de Nicotiana tabacum está relacionado a deterrência na alimentação das larvas de S. zeamais.
\end{abstract}

Palavras-chave: estratégias de controle; plantas bioativas; Sitophilus zeamais.

\section{Reduction of insect feeding and oviposition of Sitophilus zeamais Motschulsky (Coleoptera: Curculionidae) by post-vegetables used in stored maiz}

\begin{abstract}
Plants with insecticidal effect are among the alternatives as a tool to control the insect-pest of stored grains. The objective of this study was to characterize the efficiency of plant-based powders on Sitophilus zeamais insects in stored grains. Using fourteen plant species (Arnica montana Baccharis trimera, Coriandrum sativum, Coffea arabica, Cymbopogon martini, Helianthus annuus, Lippia gracillis, Mentha pulegium, Moringa oleifera, Nicotiana tabacum, Piper aduncum, Piper hispidinervum, Sesamum indicum and Vitis labrusca) obtained from tritured vegetable material, and compared with a control treatment. The experiment carried out in a completely randomized design, with six replicates each treatment. In the tests, $0.5 \mathrm{~g}$ of the inert powders mixed into the grains $(50 \mathrm{~g})$ of hybrid corn AS 1551 into plastic vials and infested with 10 emerged adult of $S$. zeamais. After 20 days of infestation, oviposition evaluated and counts of emerged adults. At the end of the infestation period, evaluated the dry weight of the insects, the weight of grains consumed and the period of development (egg-adult). Most of the vegetal powders presented effective action on the emerged insects. By the use of the powders, it verified reduction in the ooposition, except for Vitis labrusca and Sesamum indicum whose egg numbers were higher than control, also had less effect on the development of caruncho larvae. The low weight of adults from corn kernels treated with Nicotiana tabacum powder is related reduction in feeding of $S$. zeamais larvae.
\end{abstract}

Keywords: control strategies; bioactive plants; Sitophilus zeamais. 


\section{Introdução}

A pós-colheita do milho é um conjunto de processos, cuja dinâmica da demanda conhecimento, tecnologia e práticas preventivas específicas. Tratamentos com fosfina, inseticidas líquidos permitem um eficiente controle de insetos-praga em grãos de armazenados, sob condições não controladas, mantendo a qualidade fisiológica (LIMA JÚNIOR, 2012; DIAS; RAUTA; WINCK, 2017).

O Sitophilus zeamais Motschulsky (Coleoptera: Curculionidae) considerado uma praga primária interna, pode apresentar infestação cruzada, ou seja, infestar tanto as sementes no campo quanto no armazém (LORINI et al., 2015), por meio de ovos, larvas ou adultos, que apresentam ciclo de vida de 34 dias (ovoadulto) e incubação de 3-6 dias (BRITO, 2015), com potencial de danos em grãos armazenados de $10 \%$ ao ano (LORINI, 2015). Aspectos relacionados a toxicidade de inseticidas, insetos com resistência a produtos químicos, riscos à saúde e contaminações, e custos para controle (NWOSU et al., 2015; CAMPOS et al., 2013; JAIROCE et al., 2016; DOGAN et al., 2017) têm incentivado o desenvolvimento de pesquisas com inseticidas vegetais como estratégia de controle alternativo e sustentável de pragas (CORRÊA; SALGADO, 2011; GONÇALVES et al., 2015; MATEUS et al., 2017; FERREIRA; NASCIMENTO; SILVA, 2017; BARDIN et al., 2015).

Das substâncias úteis para o controle de insetos em plantas ou grãos destacam-se as de ação esterilizadora, que atuam como inseticida repelentes, que apenas afastam os insetos, ou inibidores da alimentação (CORRÊA; SALGADO, 2011). A proteção pode ser mais interessante com substâncias que desestimulem a ação dos herbívoros. A eliminação de alguns insetos pode causar desequilíbrio do sistema ecológico (ARAÚJO et al., 2018; CAMPOS et al., 2016).

Produtos vegetais (extratos, pós e óleos vegetais) utilizado no controle de insetos, apresentam como característica a baixa toxicidade a organismos não alvos, sendo biodegradáveis e em alguns casos seletivos (BOTTI et al., 2015; SAAD et al., 2017), geralmente são de baixo custo e fácil aquisição. $A$ ação ocorre por contato, ingestão e fumigação, provocam mortalidade, repelência, efeitos no crescimento, oviposição e emergência de adultos (CAMPOS et al., 2014; SANTOS; RAMALHO; PÁDUA, 2018; SCOPEL et al., 2018), quando observadas as potenciais propriedades inseticidas (FABRO; RABELO; GRISA, 2014; GONÇALVES et al., 2015; RIBEIRO et al., 2016a; RIBEIRO et al., 2016b).

Os constituintes dos extratos, pós e óleos essenciais (OE's) têm aumentado o emprego de vegetais e de produtos no controle de insetos (KRINSKI; MASSAROLI; MACHADO, 2014). Em condições de laboratório, OE de cravo-da-índia nas concentrações de 17,9 e $35 \mu \mathrm{L} \mathrm{g}^{-1}$ causou $100 \%$ de mortalidade dos insetos $S$. zeamais e $A$. obtectus após $48 \mathrm{~h}$ (JAIROCE et al., 2016), mas a exposição ao pó não foi testada. A ação de características tóxicas, repelentes, deterrentes de alimentação e oviposição, inibidoras de crescimento, formam uma defesa química contra insetos e outros organismos fitófagos (SAAD et al., 2017).

Para o manejo integrado de pragas de grãos armazenados, óleo essencial de Alfazema (CRUZ et al., 2012) de Cymbopongon citratus e Zingiber officcinale (FRANZ; KNAAK; FIUZA, 2011) e de Schinus molle (FERNANDES; FAVERO, 2014) apresentaram ação repelente sobre o Sitophilus zeamais. A atividade inseticida do pó $(0,125 \mathrm{~g})$ de Chenopodium ambrosioides (LIMA-MENDONÇA et al., 2013) causou mortalidade total de $S$. zeamais no milho armazenado. Enquanto o pó à base de cinza de xisto, cinza de madeira e terra diatomácea foi eficiente no controle de $S$. zeamais a partir de trinta dias de armazenamento (SILVA et al., 2012), o pó de Eucalyptus citriodora provocou maior repelência em milho armazenado (PROCÓPIO et al., 2003). Expostos ao pó de crambe por 15 dias, Siqueira e Simonetti (2015) constataram $97,5 \%$ de mortalidade de adultos, mas, sua ação sobre o inseto vivo nos grãos mostrou-se inferior à formulação dos extratos aquosos de crambe (SIMONETTII, 2016). Extrato de nim (Azadirachta indica) apresentou eficiência no controle de Zabrotes subfasciatus, Acanthoscelides obtectus e Sitophilus zeamais em grãos de feijão e milho armazenados (WENNECK et al., 2019).

Quanto a utilização do pó de fumo no manejo de pragas, estudos indicam sua aplicação no controle de insetos-praga no campo, como à eliminação de pulgões, brocas. No entanto, por pertencer à mesma família, plantas solanaceae não podem ser submetidas ao uso de pó de fumo, devido a transmissão do vírus "mosaico do fumo" (ECOTELHADO, 2019), associado a efeitos fitotóxicos sobre insetos-praga em grãos 
armazenados, a área carece de dados científicos. À cerca da eficiência no controle de insetos-praga em hortaliças, a nicotina substância proveniente do extrato de fumo, em função da hiperexcitação no sistema nervoso levou o inseto à morte (SILVA et al., 2017).

Diante do exposto, essa pesquisa teve como objetivo avaliar a atividade inseticida de pós de origem vegetal na mortalidade, número de ovos viáveis, emergência de Sitophilus zeamais e índice de repelência/derretência em grãos de milho armazenados.

\section{Material e métodos}

O Experimento foi conduzido em propriedade rural de base familiar, situada a 250 m de altitude nas coordenadas latitude $29^{\circ} 42^{\prime} 47^{\prime \prime}$ Sul e longitude $52^{\circ} 25^{\prime} 54^{\prime \prime}$ Oeste, no período 2017/2018. Para equilíbrio higroscópico da umidade e eliminação de infestações do milho híbrido AS 1551 recém-colhido, os grãos para a criação e bioensaios com $S$. zeamais, acondicionados em saco plástico após permanência de sete dias em freezer $\left(-10^{\circ} \mathrm{C}\right)$, foram mantidos à temperatura ambiente por dez dias. Para a realização dos ensaios foi mantida uma criação estoque de $S$. zeamais no interior de câmara tipo BOD climatizada à $25 \pm 2{ }^{\circ} \mathrm{C}$, UR $70 \pm 10 \%$ e fotofase de $12 \mathrm{~h}$.

$\mathrm{Na}$ criação dos insetos foram utilizados frascos de vidro (1 L) fechado com tampa perfurada revestida com tela (30 mesh) permitindo a circulação de ar. Cada frasco recebeu 0,5 kg de grãos de milho híbrido AS 1551 recém-colhidos, sendo infestado com 300 adultos de $S$. zeamais, não sexados, que após o período de postura de 15 dias, foram retirados e os recipientes estocados até a emergência da geração F1.

A partir dos insetos provenientes da criação, foram preparados 14 tratamentos utilizando o pó vegetal obtido da parte área da planta (caule, folhas, flores, inflorescência, frutos, sementes) das espécies Nicotiana tabacum (Tabaco), Moringa oleifera (moringa), Coffea arabica (Café), Helianthus annuus (girassol), Vitis labrusca (uva), Sesamum indicum (gergelim); Lippia gracillis (alecrim), Cymbopogon martini (Palma-rosa), Piper aduncum (Pimenta-demacaco), Piper hispidinervum (pimenta longa), Arnica montana (Arnica), Baccharis trimera (Carqueja), Coriandrum sativum (Coentro) e Mentha pulegium (Poejo). As plantas colhidas em propriedade familiar e áreas vizinhas à propriedade no período janeiro/junho 2017, passaram por triagem para a remoção de impurezas e lavagem. Após, a matéria-prima (caule, folhas, flores, inflorescência, frutos, sementes) com teor de água de $60-80 \%$ em base seca (bs) foi espalhada em camadas finas sobre tela e expostas em terreiro suspenso até $8 \pm 1 \%$. A matéria-prima (caule, folhas, flores, inflorescência, frutos, sementes) das espécies vegetais recém-seca foi triturada em moinho de facas, na sequência em moinho de bolas por cinco minutos para padronização da granulometria e peneirado (malha $2 \mathrm{~mm}$ ) para obtenção do pó, que foi acondicionado em tubos falcon e armazenados em freezer a temperatura $10^{\circ} \mathrm{C}$ até sua utilização. O teor de água foi determinado em estufa com circulação de ar forçado $\left(105^{\circ} \mathrm{C} / 24 \mathrm{~h}\right)$.

Foram realizados testes preliminares com doses crescentes de pó e massa de grãos (50 g), pesados em balança analítica de precisão $( \pm 0,01$ g), e a partir da curva de calibração determinado a dose de pó utilizada nos bioensaios. Para avaliar a eficiência dos pó-vegetais sobre o inseto $S$. zeamais em condições não controladas, o experimento foi constituído de 14 bioensaios ( 50 $\mathrm{g}$ de grãos $+0,5 \mathrm{~g}$ de pó) mais o bioensaio testemunha (50 g de grãos), conduzido em delineamento inteiramente casualizado, com seis repetições por tratamento. A pesagem massa de cada material foi verificada.

Cada unidade experimental, em frasco plástico $(250 \mathrm{~mL})$ com tampa perfurada, permitindo trocas gasosas com o exterior, constituída por $50 \mathrm{~g}$ de grãos de milho misturados a $0,5 \mathrm{~g}$ dos respectivos pós inertes, foi infestada por 20 adultos, não sexados e com idade 10-20 dias, emergidos de criação de $S$. zeamais descrita anteriormente. Decorridos cinco dias do confinamento, efetuou-se a contagem dos insetos vivos e mortos. Aos 25 dias após a infestação contabilizou-se a quantidade de ovos em cada unidade experimental, calculando-se o índice de preferência para oviposição $\left(\mathrm{I}_{\mathrm{po}}\right)$ conforme por Baldin et al. (2001). Os adultos emergidos foram quantificados, pesados $e$ descartados em avaliações diárias, a partir do 350 dia do confinamento até o término da emergência (cinco dias consecutivos sem emergência), determinando-se o período de desenvolvimento (ovo-adulto) e massa seca de grãos consumidos por $S$. zeamais

Paralelo a sobrevivência, para a avaliação da progênie 1 e danos nos grãos, decorridos 10 
dias da infestação, os adultos foram retirados e as unidades amostrais mantidas em condições climáticas não controladas por 60 dias para a contagem de adultos emergidos (avaliações diárias até cinco dias consecutivos sem emergência) e danos em decorrência da alimentação de $S$. zeamais pela avaliação visual do grão e a perda de peso pela Equação $\mathrm{Pp}=$ [(número de grãos danificados / número total de grãos)* 0,125] proposta por Adams e Schulten (1976).

Quanto à atratividade de adultos de $S$. zeamais a avaliação dos efeitos de pós vegetais através do teste com livre chance de escolha em arenas constituídas de 14 recipientes plásticos, simetricamente conectados a um recipiente central por meio de tubos plásticos de mesmo comprimento sobre uma base acrílica com diâmetro de $40 \mathrm{~cm}$. Em um dos recipientes colocou-se $50 \mathrm{~g}$ de grãos de milho não tratado, enquanto que nos demais, aleatoriamente foram colocadas amostras de 50 grãos com $0,5 \mathrm{~g}$ dos respectivos pós. No recipiente central foram liberados 50 adultos de $\mathrm{S}$. zeamais, não sexados e com idade entre 10 e 20 dias. Decorridos 24 h, avaliou-se os insetos em cada recipiente e recolocados nas arenas, onde permaneceram por mais quatro dias.

Os diversos tratamentos foram comparados entre si por meio do índice de repelência (IR) ou derretência (ID) pela Equação IR/ID = [\% de insetos nos grãos tratados com pó / (\% de insetos nos grãos tratados com pó $+\%$ de insetos nos grãos não tratados)] adaptada de Lin; Kogan; Fischer (1990). Para as médias dos tratamentos o intervalo de classificação (IC) foi calculado pela Equação $I C=1 \pm t\left(n^{\circ}\right.$ de repetições $\times 0,05) \times\left(D P / \sqrt{ } n^{\circ}\right.$ repetições $) . ~ N a$ avalição os pós foram considerados neutros quando o valor de ID esteve compreendido dentro do IC; Repelente/derretente quando o valor do IR/ID foi inferior ao menor do valor obtido para o IC; e atraente e/ou fagodeterrente quando o IR/ID foi superior ao maior IC calculado.

Ao final dos ensaios, por meio de comparação entre peso dos grãos infestadas e peso dos grãos não infestadas determinou-se consumo de grãos pelas larvas de $S$. zeamais. Os dados foram submetidos à análise de variância pelo teste de Tukey $(p \leq 0,05)$, conforme metodologia indicada por Ferreira (2014), utilizando-se o programa estatístico software SISVAR.

\section{Resultados e discussão}

Considerando o tempo de exposição e a concentração de pó utilizada, as espécies vegetais testadas mostraram diferentes respostas. Em razão dos valores observados, verificou-se que a aplicação de $0,5 \mathrm{~g}$ de pó vegetal aos grãos de milho influencia na mortalidade de insetos adultos e, em sua maioria, a ação do pó seco apresentou resultados efetivos na redução da postura ( $\left.I_{P O}\right)$, na emergência e no período de desenvolvimento (ovo-adulto) de $S$. Zeamais (Tabela 1). Scopel et al. (2018) observaram que o pó de Pipper nigrum apresentou propriedade inseticida sobre adultos de S. zeamais. Santos; Ramalho; Pádua (2018) relataram que o pó proveniente de $A$. indica, $P$. nigrum e Sizygyum aromaticum apresentam potencial inseticida no controle de Zabrotes subfasciatus. 
Tabela 1. Índice de oviposição ( $\left.I_{P o}\right)$, adultos emergidos ( $\left.A E\right)$, peso seco de adultos (PSA) e peso de grãos consumido (PGC) por S. zeamais tratados com pós inertes de espécies vegetais.

\begin{tabular}{|c|c|c|c|c|c|}
\hline \multirow{2}{*}{ Tratamento $^{1}$} & \multirow{2}{*}{$\mathrm{IPO}^{2}$} & \multirow{2}{*}{ Adultos emergidos } & PSA & PGC & Ovo-Adulto \\
\hline & & & (mg) & (g) & (dias) \\
\hline Controle & 0,462 & $99, \pm 7,2^{a}$ & $2,66 \pm 0,32^{a}$ & $1,816^{a}$ & $35^{a}$ \\
\hline Nicotiana tabacum & $-82,973$ & $0,5 \pm 0,2^{c}$ & $0,86 \pm 0,03^{d}$ & $0,034^{d}$ & $21^{\mathrm{e}}$ \\
\hline Baccharis trimera & $-67,535$ & $3,2 \pm 0,2^{b}$ & $1,12 \pm 0,12^{c}$ & $0,116^{c}$ & $28^{c}$ \\
\hline $\begin{array}{l}\text { Coriandrum } \\
\text { sativum }\end{array}$ & $-41,685$ & $3,4 \pm 0,1^{b}$ & $2,17 \pm 0,06^{b}$ & $0,356^{b}$ & $28^{c}$ \\
\hline Mentha pulegium. & $-31,598$ & $4,5 \pm 0,2^{b}$ & $2,14 \pm 0,09^{b}$ & $0,309^{b}$ & $31^{b}$ \\
\hline Coffea arabica & $-63,283$ & $2,2 \pm 0,5^{b}$ & $1,25 \pm 0,02^{c}$ & $0,106^{c}$ & $25^{d}$ \\
\hline $\begin{array}{l}\text { Cymbopogon } \\
\text { martini }\end{array}$ & $-63,498$ & $3,1 \pm 1,2^{b}$ & $1,94 \pm 0,07^{b}$ & $0,121^{c}$ & $30^{b}$ \\
\hline Piper aduncum & $-66,283$ & $2,1 \pm 1,1^{b}$ & $1,83 \pm 0,03^{b}$ & $0,128^{c}$ & $30^{b}$ \\
\hline Piper hispidinervum & $-58,338$ & $3,1 \pm 0,3^{b}$ & $1,77 \pm 0,02^{b}$ & $0,124^{c}$ & $31^{b}$ \\
\hline Lippia gracillis & $-63,202$ & $3,3 \pm 0,2^{b}$ & $2,14 \pm 0,04^{b}$ & $0,138^{c}$ & $28^{c}$ \\
\hline Vitis labrusca & 2,519 & $80,2 \pm 2,5^{a}$ & $2,79 \pm 0,18^{a}$ & $1,840^{a}$ & $36^{\mathrm{a}}$ \\
\hline Moringa oleífera & $-25,378$ & $3,1 \pm 0,3^{b}$ & $1,59 \pm 0,09^{b}$ & $0,114^{c}$ & $28^{\mathrm{c}}$ \\
\hline Sesamum indicum & 1,403 & $79,0 \pm 1,5^{a}$ & $2,87 \pm 0,22^{a}$ & $1,780^{a}$ & $36^{\mathrm{a}}$ \\
\hline Arnica montana & $-27,042$ & $4,9 \pm 0,7^{b}$ & $1,97 \pm 0,08^{b}$ & $0,380^{b}$ & $28^{c}$ \\
\hline Helianthus annuus & $-39,245$ & $3,3 \pm 0,2^{b}$ & $2,09 \pm 0,07^{b}$ & $0,346^{b}$ & $28^{c}$ \\
\hline
\end{tabular}

${ }^{1}$ Dados originais. ${ }^{2}$ para análise as médias foram transformadas em $(X+0,5)^{1 / 2}$. Médias seguidas de mesma letra não diferem entre si pelo teste Tukey $(p \leq 0,05)$.

Os tratamentos contendo pós de $N$. tabacum, B. trimera, M. pulegium, C. arabica e C. sativum foram os menos ovipositados por $S$. zeamais, diferindo de $V$. labrusca e $S$. indicum que apresentaram a maior média de ovos, tanto ovos por parcela como por grão, logo, a menor oviposição diminui a infestação gradativamente. Além de reduzir a ovoposição, estudos desenvolvidos por Araújo et al. (2016) concluíram que o extrato de $N$. tabacum inviabilizam o desenvolvimento de ovos de Rhipicephalus microplus, com eficiência para uso na pecuária independentemente do método de extração. Extratos botânicos e óleos essenciais associados à nanotecnologia oferece um potencial considerável para o aprimoramento da qualidade e segurança dos alimentos (LOPES et al., 2017), evitando a rejeição de alimentos embalados causada por insetos (RAMPELOTTI-FERREIRA et al., 2017; PRASAD; PANDEY; BARMAN, 2016).

Os valores do $I_{P O}$ evidenciam que o pó $N$. tabacum foi mais eficaz para o controle de $S$. zeamais (Tabela 1). Todos os pós vegetais utilizados influenciaram no período de desenvolvimento do S. zeamais, com exceção de V. labrusca e S. indicum, cuja sobrevivência foi superior a 36 dias, não diferenciando do tratamento controle. $\mathrm{O}$ baixo peso dos insetos adultos nos grãos tratados com os pós vegetais sugere algum efeito prejudicial relacionada às diferentes de substâncias secundárias presentes nas distintas partes da planta sobre as larvas do inseto (RIBEIRO et al., 2016b; RIBEIRO et al., 2016b).

No período das avaliações, em relação aos parâmetros biológicos, devido à ausência de insetos mortos, o tratamento controle não apresentou mortalidade natural, enquanto a atuação dos pós vegetais reduziu o número de ovos, adultos emergidos, período de desenvolvimento e peso de grãos indicando efeito/ação sobre o inseto. No controle de pragas agrícolas, o potencial de aplicações dessas substâncias no manejo de pragas e vetores (SILVA; SATO; BAGA, 2019) atribui-se a defesa química contra insetos (SAAD et al., 2017).

Os pós vegetais misturados aos grãos provavelmente afetaram a viabilidade dos ovos ou a fase larval de $S$. zeamais, uma vez que a diferença estatística observada entre os materiais quanto à oviposição se manteve na avaliação de emergências (Tabela 1). Estudando a propriedade inseticida de diferentes espécies vegetais para o controle $S$. zeamais em grãos de milho híbrido AS 1555 YG e AS 1551 YG, Scopel et al. (2018) observaram uma mortalidade de $49 \%$ utilizando o pó da pimenta-do-reino (Piper nigrum); a repelência sobre gorgulhos adultos, foi de $31 \%$ 
para a macela (Anthemis sp.) e 26,5\% para Piper nigrum nos grãos do híbrido AS 1555 YG e no hibrido AS 1551 YG apenas a pimenta-do-reino demonstrou efeito repelente, enquanto o pó de coentro (Coriandrum sativum) se mostrou neutro em todos os tratamentos.

Utilizando óleos essenciais Jairoce et al. (2016), também constataram efeitos no crescimento, redução na oviposição e na emergência de insetos adultos em grãos de milho armazenados. Campos et al. (2014) com óleo essencial e Baldin et al. (2009) utilizando pós vegetais observaram resultado semelhante sobre insetos em grãos de feijão. Para o controle de Zabrotes subfasciatus em grãos de feijão fava, a dose dos pós vegetais não teve influência sobre as fases pupal/larval do inseto e para as diferentes espécies vegetais, o índice de morte prematura e de emergência de adultos de $Z$. subfasciatus foi igual para os pós testadas (SANTOS; RAMALHO; PÁDUA, 2018). Mesmo em doses baixas, Brito et al. (2015) obtiveram mortalidade de adultos, deterrência na ovoposição e redução na geração $F 1$ de $Z$. subfasciatus em feijão-comum armazenado tratado com óleo essencial de Ocimum basilicum.
Esses resultados incentivam sua utilização produtos de origem vegetal como alternativas para o controle de $S$. zeamais em grãos armazenados, beneficiando diferentes sistemas de produção, especialmente, agricultura familiar e orgânicos. Por tratar-se de plantas de fácil cultivo, essas três espécies apresentam-se como alternativas viáveis no controle do gorgulho do milho. Novas pesquisas são necessárias para determinação de proporções eficientes de pós vegetais em função da massa de grãos armazenados, análise de resíduos tóxicos e para identificação molecular dos componentes nocivos aos insetos.

A ação repelente no controle de pragas de grãos armazenados está associada a composição da espécie vegetal. Existe uma tendência de quanto maior a repelência, menor será a infestação, resultando na redução supressão da postura e, logo, do número de insetos emergidos. Observou variação no efeito dos pós vegetais sobre adultos de $S$. zeamais (Tabela 2).

Tabela 2. Porcentagem de insetos atraídos, índice de repelência/derretência, intervalo de classificação e classificação de pós vegetais, na dose de $0,5 \mathrm{~g} \mathrm{~g} \mathrm{~g}^{-1}$ de grãos de milho, sobre adultos de $S$. zeamais. Teste de livre chance de escolha em condições não controladas.

\begin{tabular}{|c|c|c|c|c|c|}
\hline & \multicolumn{2}{|c|}{ Adultos atraídos $^{1}$} & \multirow{2}{*}{ IR ou ID } & \multirow{2}{*}{$\mathrm{IC}^{3}$} & \multirow{2}{*}{ Classificação } \\
\hline Tratamento & Controle & Pó vegetal & & & \\
\hline Controle $^{4}$ & $92,7^{a}$ & $7,3^{d}$ & $0,85 \pm 0,66^{a}$ & $1 \pm 0,14$ & Repelência/Derretência \\
\hline Nicotiana tabacum & $99,7^{\mathrm{a}}$ & $0,3^{d}$ & $0,99 \pm 0,04^{a}$ & $1 \pm 0,03$ & Repelência/Derretência \\
\hline Baccharis trimera & $82,3^{b}$ & $17,7^{c}$ & $0,64 \pm 0,16^{b}$ & $1 \pm 0,23$ & Repelência/Derretência \\
\hline Coriandrum sativum & $77,4^{b}$ & $22,6^{c}$ & $0,54 \pm 0,18^{b}$ & $1 \pm 0,57$ & Repelência/Derretência \\
\hline Mentha pulegium & $71,9^{b}$ & $28,1^{c}$ & $0,43 \pm 0,22^{b}$ & $1 \pm 0,55$ & Repelência/Derretência \\
\hline Coffea arabica & $96,9^{a}$ & $3,1^{d}$ & $0,93 \pm 0,09^{a}$ & $1 \pm 0,06$ & Repelência/Derretência \\
\hline Cymbopogon martini & $80,9^{b}$ & $19,1^{c}$ & $0,61 \pm 0,32^{b}$ & $1 \pm 0,33$ & Repelência/Derretência \\
\hline Piper aduncum & $96,9^{a}$ & $3,1^{d}$ & $0,93 \pm 0,09^{b}$ & $1 \pm 0,08$ & Repelência/Derretência \\
\hline Piper hispidinervum & $84,3^{b}$ & $16,7^{c}$ & $0,68 \pm 0,33^{b}$ & $1 \pm 0,31$ & Repelência/Derretência \\
\hline Lippia gracillis & $31,2^{c}$ & $69,8^{b}$ & $-0,37 \pm 0,21^{d}$ & $1 \pm 0,86$ & Atraente \\
\hline Vitis labrusca & $16,8^{d}$ & $83,2^{a}$ & $-0,66 \pm 0,33^{d}$ & $1 \pm 0,88$ & Atraente \\
\hline Moringa oleífera & $82,6^{b}$ & $17,4^{\mathrm{c}}$ & $0,65 \pm 0,22^{b}$ & $1 \pm 0,31$ & Repelência/Derretência \\
\hline Sesamum indicum & $31,5^{c}$ & $68,5^{b}$ & $-0,37 \pm 0,55^{d}$ & $1 \pm 0,86$ & Atraente \\
\hline Arnica montana & $64,7^{b}$ & $35,3^{c}$ & $0,29 \pm 0,47^{c}$ & $1 \pm 0,45$ & Repelência/Derretência \\
\hline Helianthus annuus & $81,4^{b}$ & $18,6^{\mathrm{c}}$ & $0,62 \pm 0,28^{b}$ & $1 \pm 0,29$ & Repelência/Derretência \\
\hline Desvio Padrão & 25,91 & 25,99 & 0,5182 & & \\
\hline
\end{tabular}


A classificação de acordo com a atração de $S$. zeamais, mostrou que pó vegetal de gergelim (S. indicum), uva (V. labrusca) e alecrim (L. gracillis) foram atrativos, com porcentagem de adultos superior ao controle, possivelmente relacionado a ação terpênica de compostos secundários (MORAIS et al., 2016). A atratividade pode, também, ser variável em uma espécie devido a fatores genéticos. Proteção contra pragas e consumo variável por insetos Maia et al. (2018) atribuíram aos genótipos testados.

O tratamento contendo pó de $N$. tabacum resultou em maior ação sobre os insetos expostos, fazendo com que paralisassem temporariamente sua alimentação (fagodeterrência), indicado pelo menor número de insetos nos recipientes (Tabela 2), menor peso seco de adultos e menor consumo de massa (Tabela 1). Corroborando os índices de Ipo positivos obtido para grãos com pó de uva e de gergelim, que mais atraíram $S$. zeamais (Tabela 2 ), apresentaram maior número de adultos, peso seco adulto e massa de grão consumida (Tabela 1), portanto, sem eficácia no controle. Embora classificada como atraente, a A. montana apresentou resultado de Ipo (Tabela 1) semelhante $(p \leq 0,05)$ aos demais pós com efeito repelente/deterrente. Indicativo da formação de defesa química contra insetos fitófagos (SAAD et al., 2017; SILVA; SATO; BAGA, 2019).

Considerando a viabilidade de compostos bioativos obtidos de plantas para o controle de pragas de grãos armazenados, a repelência/deterrência do pó de $N$. tabacum (Tabela 2) associa-se aos alcaloides naturais (nicotina), que devido a rápida ação neurotóxica, principalmente por contato (MORAIS et al., 2016), levando-o à morte (SILVA et al., 2017), ou imobiliza sua atuação de (DOGAN et al., 2017), mantendo tecidos vegetais sem alterações deletérias (ARAÚJO et al., 2018; RIBEIRO; VENDRAMIM, 2017; RIBEIRO et al., 2016a). No campo, extrato aquoso do pó de tabaco (10\% (p $\mathrm{v}^{-1}$ ) apresentou eficácia similar a um inseticida sintético à base de deltametrina no controle de insetos-praga de cultivos hortícolas (RIBEIRO et al., 2016b).

Os resultados da atividade inseticida do pó de tabaco sobre $S$. zeamais indicam uma destinação interessante para o resíduo resultante do processamento das folhas de N. tabacum póscolheita. A utilização de compostos vegetais com ação tóxica, de repelência, deterrentes de alimentação e oviposição, inibidoras de crescimento pode ser uma eficiente ferramenta no controle de insetos de grãos armazenados, em especial de $S$. zeamais em milho. Porém, o potencial de aplicações dessas substâncias no manejo de pragas e vetores, exige a identificação dos compostos responsáveis pela ação deterrente, que aliado a escassez de informações no controle de pragas agrícolas em grãos armazenados, direciona o foco de novas pesquisas para a aplicação da tecnologia.

\section{Conclusões}

Grãos de milho tratados com pó inerte de N. tabacum, C. arabica e B. trimera, apresentam deterrência na alimentação das larvas de $S$. zeamais, com baixo número de adultos emergidos, peso de adultos e consumo de grãos.

O pó inerte de $V$. labrusca e $S$. indicum não apresentou efeito inseticida sobre $o S$. zeamais em grãos de milho armazenado.

\section{Referências}

ADAMS, J. M.; SCHULTEN, G. G. M. Losses caused by insects, mites and microorganisms. In: Postharvest grain loss assessment methods. Slough, England: American Association of Cereal Chemists, 1976. p.83-93.

ARAÚJO, É. A. S. G. O.; FERNANDES, S. D. C.; ROQUE, F.; DELGADO, M. N. Levantamento de plantas fitossanitárias utilizadas no manejo de pragas agrícolas. Revista Brasileira de Agroecologia, v.13, n.4, p.163-174, 2018.

ARAÚJO, I. M.; BORGES, M.; SILVA, N. P.; VILELA, H. C.; FRÁGUAS, R. M.; ABEL, I. ATIVIDADE ACARICIDA DE Nicotiana tabacum SOBRE OVOS DE Rhipicephalus (Boophilus) microplus. Acta Veterinaria Brasilica, v.10, n.2, p.190-193, 2016. https://doi.org/10.21708/avb.2016.10.2.5576

BALDIN, E.L.L., TOSCANO, L.C., LIMA, A.C.S., LARA, F.M., BOIÇA JÚNIOR, A.L. Preferência para oviposição de Bemisia tabaci Biótipo "B" por genótipos de Cucurbita moschata e Cucurbita maxima. Boletín de Sanidad Vegetal Plagas, v.26, p.409-413, 2001.

BALDIN, E.L.L.; PRADO, J.P.M.; CHRISTOVAM, R.S.; DAL POGETTO, M.H.F.A. Uso de Pós de Origem Vegetal no Controle de Acanthoscelides obtectus Say (Coleoptera: Bruchidae) em Grãos de 
Feijoeiro. BioAssay, v.4, n.2, 2009. https://doi.org/10.14295/BA.v4.0.23

BARDIN, M.; AJOUZ, S.; COMBY, M.; LOPEZFERBER, M.; GRAILLOT, B.; SIEGWART, M.; NICOT, P. C. Is the efficacy of biological control against plant diseases likely to be more durable than that of chemical pesticides? Frontiers in Plant Science, v.6, p.1-14, 2015. https://doi.org/10.3389/fpls.2015.00566

BOTTI, J. M. C.; HOLTZ, A. M.; PAULO, H. H.; FRANZIN, M. L.; PRATISSOLI, D.; PIRES, A. A. Controle alternativo do Brevicoryne brassicae (Hemiptera: Aphididae) com extratos de diferentes espécies de plantas. Revista Brasileira de Ciências Agrárias, v.10, n.2, p.178-183, 2015. https://doi.org/10.5039/agraria.v10i2a3520

BRITO, S. M. Manejo de Sitophilus zeamais em milho doce através da resistência hospedeira por antixenose e antibiose. 2015. Monografia Faculdade de Agronomia e Medicina Veterinária, Universidade de Brasília, Brasília, 2015.

BRITO, S. S. S.; MAGALHÃES, C. R. I.; OLIVEIRA, C. R. F.; OLIVEIRA, C. H. C. M.; FERRAZ, M. S. S.; MAGALHÃES, T. A. Bioatividade de óleos essenciais sobre Zabrotes subfasciatus Boh. (Coleoptera: Chrysomelidae) em feijão-comum armazenado. Revista Brasileira de Ciências Agrárias, v. 10, n. 2, p. 243-248, 2015. https://doi.org/10.5039/agraria.v10i2a5316

CAMPOS, S. C.; SILVA, C. G.; CAMPANA, P. R. V.; ALMEIDA, V. L. Toxicidade de espécies vegetais. Revista Brasileira de Plantas Medicinais, Campinas, v.18, n.1 (suplemento I), p.373-382, 2016. https://doi.org/10.1590/1983$\underline{084 X / 15 \quad 057}$

CAMPOS, A. C. T.; RADUNZ, L. L.; RANDUZ, A. L.; MOSSI, A. J.; DIONELLO, R. G.; ECKER, S. L. Atividade repelente e inseticida do óleo essencial de carqueja doce sobre o caruncho do feijão. Revista Brasileira de Engenharia Agrícola e Ambiental, v.18, n.8, p.861-865, 2014. https://doi.org/10.1590/1807-

1929/agriambi.v18n08p861-865

CORRÊA, J. C. R.; SALGADO, H. R. N. Atividade inseticida das plantas e aplicações: revisão. Revista Brasileira de Plantas Medicinais, v. 13, n.
4, 2011. https://doi.org/10.1590/S1516$\underline{05722011000400016}$

CRUZ, C. S. A.; SOUSA, F. C.; MEDEIROS, M. B.; SILVA, L. M. M.; GOMES, J. P. Interferência de óleos essenciais na preferência de Sitophilus Zeamais (coleoptera: curculionidae) em grãos de milho. Revista Verde, Mossoró, v. 7, n. 3, p. 187193, 2012.

DIAS, S. P.; RAUTA, J.; WINCK, C. A. Condições de armazenagem e qualidade da matéria prima: estudo de caso em uma derivadora de alimentos a base de milho. Revista Produção e Desenvolvimento, v.3, n.3, p.18-33, 2017. https://doi.org/10.32358/rpd.2017.v3.246

DOGAN,M.; EMSEN, B.; AASIM, M.; YILDIRIM, E. Ceratophyllum demersum $\mathrm{L}$. extract as a botanical insecticide for controlling the maize weevil, Sitophilus zeamais Motschulsky (Coleoptera: Curculionidae). Egyptian Journal of Biological Pest Control, v. 27, n.1, p.11-15, 2017.

FABRO, J.R.; RABELO, A.K.; GRISA, F. Métodos ecológicos de controle de insetos e de doenças das plantas e dos solos. Francisco Beltrão: ASSESOAR; 2014.

ECOTELHADO. Como usar fumo nas plantas para controle de pragas. 2019. Disponível em: https://ecotelhado.com/como-usar-fumo-nasplantas-para-controle-de-pragas/. Acesso: 31 out. 2019.

FERNANDES, E. T.; FAVERO, S. Óleo essencial de Schinus molle L. para o controle de Sitophilus Zeamais Most.1855 (Coleoptera: Curculionidae) em milho. Revista Brasileira de Agroecologia. Porto Alegre, v. 9, n. 1, p. 225-231, 2014.

FERREIRA, D. F. Sisvar: a Guide for its Bootstrap procedures in multiple comparisons. Ciência e Agrotecnologia, 2014, v.38, n.2 p. 109-112, 2014. https://doi.org/10.1590/S1413$\underline{70542014000200001}$

FERREIRA, T. C.; NASCIMENTO, D. M.; SILVA, E. O. Métodos alternativos para controle de insetos- praga em sementes. Amazonian Journal of Agricultural and Environmental Sciences, v.60, n.1, p.112-119, 2017. https://doi.org/10.4322/rca.2217 
FRANZ, A. R.; KNAAK, N.; FIUZA, L. M. Toxic effects of essential plant oils in adult Sitophilus oryzae (Linnaeus) (Coleoptera, Curculionidae). Revista Brasileira de Entomologia, Curitiba, v. 55, n.1, p.116-120, 2011.

https://doi.org/10.1590/S0085-

$\underline{56262011000100018}$

GONÇALVES, G. L. P.; RIBEIRO, L. P.; GIMENES, L.; VIEIRA, P. C.; SILVA, M. F. G. F.; FORIM, M. F.; FERNANDES, J. B.; VENDRAMIM, J. D. Lethal and sublethal toxicities of Annona sylvatica (Magnoliales: Annonaceae) extracts to Zabrotes subfasciatus (Coleoptera: Chrysomelidae: Bruchinae). The Florida Entomologist, Gainesville, v.98, n.3, p.921-928. 2015. https://doi.org/10.1653/024.098.0317

JAIROCE, C.F.; TEIXEIRA, C.M.; NUNES, C.F.P.; NUNES, A.M.; PEREIRA, C.M.P.; GARCIA, F.R.M. Insecticide activity of clove essential oil on bean weevil and maize weevil. Revista Brasileira de Engenharia Agrícola e Ambiental, Campina Grande, v.20, n.1, p.72-77, 2016. https://doi.org/10.1590/1807-

1929/agriambi.v20n1p72-77

KRINSKI, D.; MASSAROLI, A.; MACHADO, M. Potencial inseticida de plantas da família Annonaceae. Revista Brasileira de Fruticultura, v. 36, edição especial, p. 225-242, 2014. https://doi.org/10.1590/S0100-

$\underline{29452014000500027}$

LIMA JÚNIOR, A.F.; OLIVEIRA, I.P.; ROSA, S.R.A.; SILVA, A.J.; MORAIS, M.M. Controle de pragas de grãos armazenados: uso e aplicação de fosfetos. Revista Eletrônica, v.5, p.180-194, 2012.

LIMA-MENDONÇA, A.; BROGLIO, S.M.F.; ARAÚJO, A.M.N.; LOPES, D.O.P.; N.S. DIAS-PINI. Efeito de pós vegetais sobre Sitophilus zeamais (Mots., 1855) (Coleoptera: Curculionidae). Arquivos do Instituto Biológico, São Paulo, v.80, n.1, p.91-97, $2013 . \quad$ https://doi.org/10.1590/S1808$\underline{16572013000100013}$

LIN, H.; KOGAN, M.; FISCHER, D. Induced resistance in soybean to the Mexican bean beetle (Coleoptera: Coccinellidae): comparisons of inducing factors. Environmental Entomology, College Park, v.19, p.1852-1857, 1990. https://doi.org/10.1093/ee/19.6.1852
LOPES, E.; NOBRE, D. A. C.; CARVALHO FILHO, A.; MENDES, F. (Eds) A química na produção vegetal. 2017. 399 p. https://www.researchgate.net/publication/3205 $\underline{59510 .}$.

LORINI, I; KRYZANOWSKI, F. C.; FRANÇA-NETO, J. B.; HENNING, A. A.; HENNING, F. A. Manejo Integrado de Pragas de Grãos e Sementes Armazenadas. Brasília: Embrapa, 2015. 84 p.

MAIA, P. S. P.; BOIÇA JUNIOR, A. L.; TINÔCO, R. S.; FARIAS, P. R. S.; RODRIGUES, N. E. L.; CRISTO, S. T. B. Preferência alimentar de Opsiphanes invirae em cultivares de palma de óleo. Amazonian Journal of Agricultural and Environmental Sciences, v.61, 2018. https://doi.org/10.22491/rca.2018.2390

MATEUS, A. E.; AZEVEDO, F. R.; ALVES, A. C. L.; FEITOSA, J. V. Potencial da Moringa oleifera como inseticida no controle de adultos de Sitophilus zeamais (Coleoptera: Curculionidae) em grãos de milho armazenados. Acta Iguazu, v. 6, n.2, p.112122, 2017.

MORAIS, L. A. S.; MARINHO-PRADO, J. S. Plantas com atividade inseticida. In: HALFELD-VIEIRA, B. A.; MARINHO-PRADO, J. S.; NECHET, K. L.; MORANDI, M. A. B.; BETTIOL, W. Defensivos agrícolas naturais: uso e perspectivas. Brasília: EMBRAPA, 2016. p.542-593.

NWOSU, L. C.; ADEDIRE, C. O.; OGUNWOLU, E. O. Screening for new sources of resistance to Sitophilus zeamais Motschulsky (Coleoptera: Curculionidae) infestation in stored maize genotypes. Journal of Crop Protection, v. 4, n.3, p.277-290, 2015.

PRASAD, R.; PANDEY, R.; BARMAN, I. Engineering tailored nanoparticles with microbes: quovadis. Nanomedicine and Nanobiotechnology, v. 8, p. 316-330, 2016. https://doi.org/10.1002/wnan.1363

PROCÓPIO, S. O.; VENDRAMIM, J. D.; RIBEIRO JÚNIOR, J. I.; SANTOS, J. B. Bioatividade de diversos pós de origem vegetal em relação a Sitophilus zeamais Mots. (Coleoptera: Curculionidae). Ciência e Agrotecnologia, Lavras, v.27, n.6, p.1231-1236, 2003. https://doi.org/10.1590/\$1413$\underline{70542003000600004}$ 
RAMPELOTTI-FERREIRA, F. T; COELHO J. R. A; PARRA, J. R. P; VENDRAMIM, J. D. Selectivity of plant extracts for Trichogramma pretiosum Riley (Hymenoptera: Trichogrammatidae). Ecotoxicology and Environm. Safety, v.138, p. 78-82, 2017. https://doi.org/10.1016/i.ecoenv.2016.12.026

RIBEIRO, L. P.; VENDRAMIM, J. D. Effects of organic plant extracts on behavior of Sitophilus zeamais Mots. (Coleoptera: Curculionidae) adults. Brazilian Journal of Agriculture, v.92, n.2, p. 186197, 2017. https://doi.org/10.37856/bja.v92i2.3223

RIBEIRO, L. P.; VENDRAMIM, J. D.; GONÇALVES, G. L. P.; ANSANTE, T. F.; GLORIA, E. M.; LOPES, J. C.; MELLO-SILVA, R.; FERNANDES, J. B. Searching for promising sources of grain protectors in extracts from Neotropical Annonaceae. Boletín Latinoamericano y del Caribe de Plantas Medicinales y Aromáticas, v.15, n.4, p.215-232, 2016.

RIBEIRO, L. P.; BIERMANN, A. C. S.; DORNELES, M. P.; VENDRAMIM, J. D. Ação de inseticidas botânicos sobre o curuquerê-da-couve. Agropecuária Catarinense, Florianópolis, v.29, n.2, p.84-89, maio/ago. 2016.

SAAD, K.A.; IDRIS, A.B.; MOHAMAD-ROFF, M.N.; Toxic, repellent, and deterrent effects of citronella essential oil on Bemisia tabaci (Hemiptera: Aleyrodidae) on chili plants. Journal of Entomological Science, v.52, n.2, p.16-52, 2017. https://doi.org/10.18474/JES16-32.1

SANTOS, V.S.V.; RAMALHO, P.R.; PÁDUA, L.E.M. Atividade inseticida de pós vegetais sobre Zabrotes subfasciatus (Boheman) (Coleoptera, Chrysomelidae, Bruchidae) em grãos de feijão fava. HOLOS, v. 07, p. 53-58, 2018. https://doi.org/10.15628/holos.2018.4395

SCOPEL, W.; SCOPEL, E.; BOTTEON, V.; ROZAGOMES, M. F. Bioatividade de macerados de Anthemis sp., Coriandrum sativum e Piper nigrum contra Sitophilus zeamais (Coleoptera: Curculionidae). Evidência - Ciência e Biotecnologia, v. 18, n. 1, p. 95-109, 2018. https://doi.org/10.18593/eba.v18i1.16872
SILVA, S. B.; SATO, S. M.E.; RAGA, A. Uso de extratos naturais no controle de insetos, com ênfase em moscas-das-frutas (Diptera: Tephritidae). Biológico, São Paulo, v.81, n. 1, p.130, 2019. https://doi.org/10.31368/19806221v81a10002

SILVA, L. M.; CALDAS, A. P.; RODRIGUES, A. L. M.; OLIVEIRA, J. S.; SIMONETTI, E. R. S. O uso do extrato de fumo (Nicotina tabacum) como alternativa para o controle de pragas em hortaliças. In: CONGRESSO INTERNACIONAL DAS AGRÁRIAS - COINTER, PDVAgro 2017. Anais [...]. p.1-9, 2017.

SILVA, J. F.; MELO, B. A.; PESSOA, E. B.; ALMEIDA, F. A. C.; GOMES, J. P. Bioatividade do extrato de Momordica charantia L. sobre Sitophilus Zeamais Motschulsky 1885 (Coleoptera: Curculionidae). Revista Verde, Mossoró, v. 7, n. 2, p 179-183, 2012.

SIMONETTI, A. P. M. M. Uso de crambe no controle de sitophilus zeamais mots. Em milho armazenado. 2017. 67 p. Tese (Doutorado em Engenharia Agrícola) - Universidade Estadual do Oeste do Paraná, Cascavel, 2016.

SIQUEIRA, H. M.; SIMONETTI, A.P.M.M. Avaliação da preferência alimentar e sobrevivência do Sitophilus sp. em grãos de Crambe abyssinica, Triticum spp e Zeamais. Revista Cultivando o Saber, (ed. esp.), p. 115-124, 2015.

WENNECK, G. S.; SAATH, R.; SÁ, N. O.; ARAÚJO, L. L.; VOLPATO, C. S. Potencial tóxico de extrato etéreo sobre insetos no manejo de feijão e milho armazenado. Journal of Agronomic Sciences, v.8, n.2, p.187-193, 2019. 